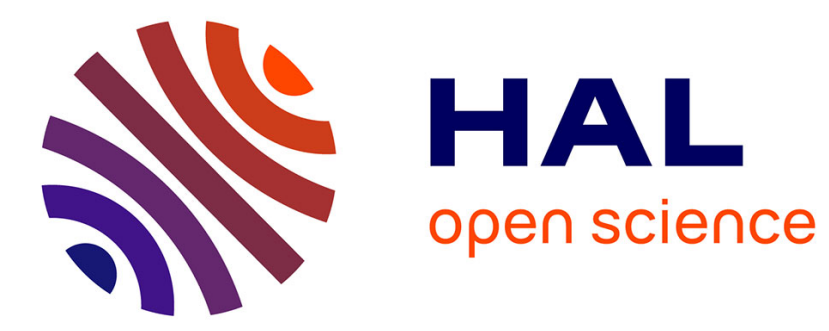

\title{
Optimal strategies for biomass productivity maximization in a photobioreactor using natural light
}

Frédéric Grognard, Andrei R. Akhmetzhanov, Olivier Bernard

\section{To cite this version:}

Frédéric Grognard, Andrei R. Akhmetzhanov, Olivier Bernard. Optimal strategies for biomass productivity maximization in a photobioreactor using natural light. Automatica, 2014, 50 (2), pp.359-368. 10.1016/j.automatica.2013.11.014 . hal-00871341

\section{HAL Id: hal-00871341 \\ https://inria.hal.science/hal-00871341}

Submitted on 6 Aug 2018

HAL is a multi-disciplinary open access archive for the deposit and dissemination of scientific research documents, whether they are published or not. The documents may come from teaching and research institutions in France or abroad, or from public or private research centers.
L'archive ouverte pluridisciplinaire $\mathbf{H A L}$, est destinée au dépôt et à la diffusion de documents scientifiques de niveau recherche, publiés ou non, émanant des établissements d'enseignement et de recherche français ou étrangers, des laboratoires publics ou privés. 


\title{
Optimal strategies for biomass productivity maximization in a photobioreactor using natural light
}

\author{
Frédéric Grognard*, Andrei R. Akhmetzhanov*, ${ }^{*}$, Olivier Bernard*, \\ ${ }^{*}$ BIOCORE, INRIA Sophia Antipolis, BP 93, \\ 06902 Sophia Antipolis Cedex, France, \\ frederic.grognard@inria.fr \\ ${ }^{\dagger}$ Institute of Evolutionary Sciences of Montpellier - \\ UMR 5554, University of Montpellier II, \\ CC065, Place Eugne Bataillon, 34090 Montpellier Cedex 5, France
}

October 9, 2013

\begin{abstract}
We address the question of optimization of the microalgal biomass long term productivity in the framework of production in photobioreactors under the influence of day/night cycles. For that, we propose a simple bioreactor model accounting for light attenuation in the reactor due to biomass density and we obtain the control law that optimizes productivity over a single day through the application of Pontryagin's maximum principle. The dilution rate is the main control, the input concentration being only used as secondary control to maintain the substrate concentration high. An important constraint on the obtained solution is that the biomass in the reactor should be at the same level at the beginning and at the end of the day so that the same control can be applied everyday and optimizes some form of long term productivity. Several scenarios are possible depending on the microalgae's strain parameters and the maximal admissible value of the dilution rate: bang-bang or bang-singular-bang control or, if the growth rate of the algae is very strong in the presence of light, constant maximal dilution. A bifurcation diagram is presented to illustrate for which values of the parameters these different behaviors occur. Finally, a simple sub-optimal bang-bang strategy is proposed that numerically achieves productivity levels that almost match those of the optimal strategy.
\end{abstract}

\section{Introduction}

Microalgae have recently received more and more attention in the frameworks of $\mathrm{CO}_{2}$ fixation and renewable energy (Huntley \& Redalje 2007, Chisti 2007). Their high actual photosynthetic yield compared to terrestrial plants (whose growth is limited by 
$\mathrm{CO}_{2}$ availability) leads to large potential algal biomass productions of several tens of tons per hectare and per year (Chisti 2007). Also, they have a potential for numerous high added value commercial applications (Spolaore, Joannis-Cassan, Duran \& Isambert 2006) as well as wastewater treatment capabilities including nutrients and heavy metal removal (Hoffmann 1998, Oswald 1988). Microalgae are cultivated at industrial scale in devices ranging from the most simple and cheap open raceways to high-tech photobioreactors. In this paper, we focus on the industrial production of microalgae in planar systems, which will be called photobioreactors by abuse of language.

The energy balance of the process is a crucial feature when dealing with bioenergy production. Thus, the only conceivable light source (i.e the primary energy source) is natural sunlight. Since it varies during the day, we have to consider this periodic feature in the design of the optimal control law. We are thus aiming at maximizing the yield of a photobioreactor operating in continuous mode with a periodic light source that has a light phase (day) and a dark phase (night). In addition to this time-variation, we will take the auto-shading in the photobioreactor into account: the pigment concentration (mainly chlorophyll) affects the light distribution and thus the biological activity within the reactor. As a consequence, for a too high biomass, light in the photobioreactor is strongly attenuated and per-capita growth is low; this also has a major impact on the productivity.

Optimal control of bioreactors has been studied for many years whether it was for metabolites production (Tartakovsky, Ulitzur \& Sheintuch 1995), ethanol fermentation (Wang \& Shyu 1997), baker yeast production (Wu, Chen \& Chiou 1985) or, more generally, optimal control of fed-batch processes taking kinetics uncertainties into account (Smets, Claes, November, Bastin \& Van Impe 2004). The control of photobioreactors is however a lot more scarce in the literature, though the influence of self-shading on the optimal setpoint (Masci, Bernard \& Grognard 2010) or on an MPC control algorithm (Becerra-Celis, Hafidi, Tebbani, Dumur \& Isambert 2008) for productivity optimization have already been studied. The light-variation was mostly absent (Masci et al. 2010, Becerra-Celis et al. 2008) or considered to be an input that could be manipulated in order to impose the physiological state of the microalgae (Marxen, Vanselow, Lippemeier, Hintze, Ruser \& Hansen 2005) or maximize productivity as one of the parameters of bioreactor design (Suh \& Lee 2003). The optimization we consider can also be classified with the Maximal Sustainable Yield problem that is classical eg. in fisheries and has been tackled in constant and some form of specific periodic environments (Clark 1990, Dong, Chen \& Sun 2007), and distincts itself from the classical periodic optimal control where periodicity was not inherent to the system (Guardabassi, Locatelli \& Rinaldi 1974). The present result is an important generalization of the result of Grognard, Akhmetzhanov, Masci \& Bernard (2010) which otherwise has not been tackled yet in the literature.

We developed a model that presents the main features of the process, with the substrate concentration in the input (marginally) and the dilution rate (mainly) as actuators for our control design. Since we want to develop a control strategy to be used on the long run, we could choose an infinite time-horizon measure of the yield. However, we rather impose the industrially relevant constraint that the control should be identical everyday and force the state of the system to be identical at the beginning of the day and 24 hours later, while optimizing a cost over one day.

The paper is structured as follows: in Section 2 we develop the photobioreactor model; in Section 3 we identify the optimal operating mode in constant light environ- 
ment; in Section 4 we develop our main result, that is the form of the optimal control law in a day \& night setting; in Section 5 we concentrate on stability issues and possible implementation methods; we conclude by a simulation study and bifurcation analysis in Section 6.

\section{A photobioreactor model with light attenuation}

Micro-algae growth in a photobioreactor is often modeled through one of two models, the Monod model (Monod 1942) or the Droop Model (Droop 1968). Here we focus on the Monod model which, though less accurate for microalgae, is more convenient for building control laws. It writes:

$$
\left\{\begin{array}{l}
\dot{S}=D\left(S_{i n}-S\right)-k \mu\left(S, I_{0}(t)\right) X, \\
\dot{X}=\mu\left(S, I_{0}(t)\right) X-D X-r X,
\end{array}\right.
$$

where $S$ and $X$ are the substrate (e.g. nitrate concentration) and biomass (measured in carbon concentration, gC. $\mathrm{L}^{-1}$ ) in the medium, which is supposed to be homogeneous through constant stirring, while $D$ is the dilution rate, $S_{i n}$ is the substrate input concentration, $\mu\left(S, I_{0}(t)\right)$ is the microalgae biomass growth rate with $I_{0}(t)$ the incoming time-varying light intensity, and $k$ is the substrate/biomass yield coefficient. Compared with the classical model, the additional $-r X$ term encompasses both biomass respiration, which has been known for a long time to impact productivity in bioreactors (see e.g. (Grobbelaar \& Soeder 1985)) and mortality, which can be quite high in the high-density reactors that will be considered.

\subsection{Modelling the growth rate}

The function $\mu($.$) is the average microalgae biomass growth rate. At any point of$ the photobioreactor, growth rate is related to local light intensity. For horizontal planar high density reactors, light is assumed to be exponentially decreasing with depth because of the auto-shading effect. We consider a constant horizontal section over the height of the reactor and vertical incoming light. Light intensity follows a BeerLambert law (Lambert 1760) where the attenuation at some depth $z$ comes from the total biomass $X z$ per surface unit contained in the layer of depth $[0, z]$ :

$$
I(X z)=I_{0} \mathrm{e}^{-a X z},
$$

where $I_{0}$ is the incident light and $a$ is a light attenuation coefficient. In microalgae, chlorophyll is mostly the cause of this shadow effect and, in model (2.1), it is best represented by a fixed portion of the biomass (Bernard, Masci \& Sciandra 2009), which yields the direct dependence in $X$ in model (2.2). With such an hypothesis on the light intensity that reaches depth $z$, higher light causes higher growth in the upper part of the reactor than in the bottom part. Supposing that light attenuation directly affects growth (Huisman, Matthijs, Visser, Balke, Sigon, Passarge, Weissing \& Mur 2002), the growth rate for a given depth $z$, when the dependency in $I$ are taken to be of Michaelis-Menten type, can then be written as

$$
\mu_{z}(S, I(X z))=\frac{I(X z)}{I(X z)+K_{I}} \mu_{S}(S) .
$$


with $K_{I}$ the associated half saturation constant and the substrate related function $\mu_{S}(S)$ is such that $\mu_{S}(0)=0$, is increasing and bounded (with $\lim _{S \rightarrow \infty} \mu_{S}(S)=\bar{\mu}$ ). Generalization for the light-related part will also be proposed later on. This results in a spatially distributed growth-rate within the reactor. However, constant stirring keeps the concentrations of $S$ and $X$ homogeneous in the reactor. Then, we can compute the mean growth rate in the reactor:

$$
\begin{aligned}
\mu\left(S, I_{0}, X\right) & =\frac{1}{L} \int_{0}^{L} \frac{I_{0} \mathrm{e}^{-a X z}}{I_{0} \mathrm{e}^{-a X z}+K_{I}} \mathrm{~d} z \mu_{S}(S) \\
& =\frac{1}{a X L} \ln \left(\frac{I_{0}+K_{I}}{I_{0} \mathrm{e}^{-a X L}+K_{I}}\right) \mu_{S}(S) .
\end{aligned}
$$

where $L$ is the depth of the reactor. This average growth rate will be used in the lumped model that we develop.

In order to determine more precisely the model, we have now to specify the varying light pattern $I_{0}(t)$. Classically, it is considered that daylight $I_{0}(t)$ varies, during the light period, as the square of a sinusoidal function. However, the introduction of such a varying light would render the computations analytically intractable. Therefore, we approximate the light source by a step function:

$$
I_{0}(t)=\left\{\begin{array}{lll}
\bar{I}_{0}, & 0 \leq t<\bar{T} \quad \text { light phase } \\
0, & \bar{T} \leq t<T \quad \text { dark phase }
\end{array}\right.
$$

with $T$ the length of the day (which will be equal to 1 if the time-unit is the day). At the equinoxes, we have that $\bar{T}=\frac{T}{2}$, but this quantity obviously depends on the time of the year. We will now provide extensions to this model so that it fits better to the production problem in high-density photobioreactors under study.

\subsection{Reduction and generalization of the model}

The system for which we want to build an optimal controller is therefore

$$
\left\{\begin{array}{l}
\dot{S}=D\left(S_{i n}-S\right)-\frac{k}{a X L} \ln \left(\frac{I_{0}(t)+K_{I}}{I_{0}(t) e^{-a X L}+K_{I}}\right) \mu_{S}(S) X \\
\dot{X}=\frac{1}{a X L} \ln \left(\frac{I_{0}(t)+K_{I}}{I_{0}(t) e^{-a X L}+K_{I}}\right) \mu_{S}(S) X-r X-D X
\end{array}\right.
$$

However, in order to maximize the productivity it is clear that the larger $S$ the better since it results in larger growth rates of the biomass $X$. Hence, the control of the inflow concentration of the substrate $S_{i n}$ should always be kept very large so as to always keep the substrate in the region where $\mu_{S}(S) \approx \bar{\mu}$. We can then concentrate on the reduced model

$$
\dot{X}=\frac{\bar{\mu}}{a L} \ln \frac{I_{0}(t)+K_{I}}{I_{0}(t) \mathrm{e}^{-a X L}+K_{I}}-r X-D X,
$$

where the only remaining control is the dilution $D$ and which then encompasses all the relevant dynamics for the control problem. As was seen in (Masci et al. 2010), the relevant concentration in the photobioreactor, with Beer-Lambert light attenuation, is not the volumic density $X$ but rather the surfacic density $x=X L$ : the evolution of this quantity is indeed independent of the reactor's depth: whether we consider a deep and very diluted reactor or a shallow high concentration reactor with identical surfacic 
density

$$
\dot{x}=\frac{\mathrm{d}(X L)}{\mathrm{d} t}=\frac{\bar{\mu}}{a} \ln \frac{I_{0}(t)+K_{I}}{I_{0}(t) \mathrm{e}^{-a x}+K_{I}}-r x-D x,
$$

which is indeed independent of the depth $L$. Model (2.4) is quite simple except for the only nonlinear term which directly comes from the form of $\mu_{I}$ and from the BeerLambert law (2.2). In order to generalize our approach to more light responses (e.g. for high density photobioreactor with possible high light inhibition, see (Bernard 2011)) and to not restrict ourselves to the Beer-Lambert law, we propose a generalized model that retains the main features of (2.4) by taking advantage of the special form of the light source: during the day of length $\bar{T}<T$, the system is written as

$$
\dot{x}=f(x)-r x-u x,
$$

with $f(x)$ the total growth and $u$ the dilution rate; during the night of length $T-\bar{T}$, the dynamics become

$$
\dot{x}=-r x-u x .
$$

In order to couple both these systems, we define $h(t)$ as a step function whose value is 1 for $t<\bar{T}$ and 0 for $t \geq \bar{T}$ that will allow to synthetize (2.5)-(2.6) in the form that is

$$
\dot{x}=f(x) h(t)-r x-u x .
$$

Assumption 1 The growth function satisfies

(i) $f: \mathbb{R}^{+} \rightarrow \mathbb{R}$ is $\mathcal{C}^{2}$, upper-bounded, satisfies $f(0)=0, f^{\prime}(0)>0, f^{\prime \prime}(x)<0$ for all $x \geq 0$

(ii) $f^{\prime}(0) \bar{T}>r T$ with $r>0$ and $T>\bar{T}>0$.

Such a choice of $f(x)$ yields the following somewhat trivial property:

Property 1 If $f(x)$ satisfies Assumption 1-(i), we have that, for any $x>0$ :

$$
f^{\prime}(0)>\frac{f(x)}{x}>f^{\prime}(x) \text { and } \frac{\mathrm{d}}{\mathrm{d} x}\left(\frac{f(x)}{x}\right)<0,
$$

which also shows that $\liminf _{x \rightarrow+\infty} f^{\prime}(x) \leq 0$.

Proof: We have $f(x)=f(0)+\int_{0}^{x} f^{\prime}(s) \mathrm{d} s$ with $f(0)=0$ and $f^{\prime}(0)>f^{\prime}(s)>$ $f^{\prime}(x)$ for all $0<s<x$ due to the concavity, so that $f(x)<\int_{0}^{x} f^{\prime}(0) \mathrm{d} s=f^{\prime}(0) x$ and $f(x)>\int_{0}^{x} f^{\prime}(x) \mathrm{d} s=f^{\prime}(x) x$. The decreasing property of $\frac{f(x)}{x}$ comes from the explicit computation $\frac{\mathrm{d}}{\mathrm{d} x}\left(\frac{f(x)}{x}\right)=\frac{f^{\prime}(x) x-f(x)}{x^{2}}$ which is negative thanks to the previous property.

Since the function $f(x)$ is upper-bounded, the ratio $f(x) / x$ tends to zero as $x \rightarrow$ $+\infty$, which means that $\liminf _{x \rightarrow+\infty} f^{\prime}(x) \leq \lim _{x \rightarrow+\infty} \frac{f(x)}{x}=0$.

The four properties that we impose on $f(x)$ should be expected from the net-growth in a photobioreactor: no growth should take place in the absence of biomass, it should be increasing because additional biomass should lead to more growth (at least at low densities); it should be bounded because, when $x$ is very large the bottom of the reactor is in the dark, so that adding new biomass simply increases the dark zone without 
allowing additional growth; and the growth-rate $\frac{f(x)}{x}$ should be decreasing because additional biomass slightly degrades the environment for all because of the shadowing it forces.

Lastly, in practice, $u$ cannot take any value: it should be positive, but also upperbounded since its value is determined by the maximum capacity of some pumps. We will then consider that $0 \leq u(t) \leq \bar{u}$ at all times (with $\bar{u}>0$ ).

\section{Biomass flow rate optimization in constant light envi- ronment}

In a constant light environment, the dynamics are strictly imposed by (2.5) and we will be able to exploit the system at a steady state. In this section, the best constant operating conditions are identified: the constant control $u$ and corresponding equilibrium value $\bar{x}(u)$, when it exists, are chosen in order to maximize the biomass flow rate at the output of the reactor. This is defined as $\max _{u} u \bar{x}(u) A$ (with $A$ the horizontal section of the reactor).

Since $u x=f(x)-r x$ at equilibrium, maximizing $u A x$ amounts to maximizing $f(x)-r x . f(x)$ being concave, this is equivalent to find the unique solution of $f^{\prime}(x)=$ $r$ when it exists. Note that if $r=0$, it has no solution: the smaller the positive constant $u$, the larger the productivity that can approach $\sup _{x>0} f(x)$ as much as desired. This case hints at difficulties arising when $r$ is very small.

Since $\lim _{x \rightarrow+\infty} f^{\prime}(x) \leq 0$ (see Property 1 ) and $f^{\prime}($.$) is a decreasing function of x$ because of the concavity of $f, f^{\prime}(x)=r$ has a positive solution if $f^{\prime}(0)>r$ is satisfied (which is a consequence of Assumption 1-(ii)). This condition is crucial since, without it, the only equilibrium of the system is $x=0$, independently of the choice of $u$. Under this assumption, the biomass density that corresponds to the maximization of the biomass flow rate in a constant light environment through constant control is

$$
x_{\sigma}=\left(f^{\prime}\right)^{-1}(r) \text { with } u_{\sigma}=\frac{f\left(x_{\sigma}\right)}{x_{\sigma}}-r>0 .
$$

so that that $f(x)-r x$ is increasing for $x<x_{\sigma}$ and decreasing for $x>x_{\sigma}$, which we will extensively use.

Taking into account the actuator constraint, if $u_{\sigma} \leq \bar{u}, u_{\sigma}$ yields the optimal productivity. Otherwise, if $u_{\sigma}>\bar{u}$, the actuator is under-dimensioned, and it can be computed that the best control is $u=\bar{u}$.

The equilibrium $\bar{x}(u)$ is a decreasing function, defined only for $u<f^{\prime}(0)-r$, because it is solution of $\frac{f(\bar{x}(u))}{\bar{x}(u)}-r=u$ with $\frac{f(x)}{x}$ decreasing from $f^{\prime}(0)$ at $x=0$. Also, $\bar{x}(u)$ is globally asymptotically stable in $\mathbb{R}_{+}$for $0<u<f^{\prime}(0)-r$; indeed, since $\frac{f(x)}{x}$ is a decreasing function of $x, \dot{x}=x\left(\frac{f(x)}{\bar{x}}-r-u\right)<0$ (resp. $>0$ ) for $x>\bar{x}(u)$ (resp. $x<\bar{x}(u)$ ), which shows global asymptotic stability of $\bar{x}(u)$. For convenience, we will define two other equilibria beyond $x_{\sigma}: \bar{x}^{0}=\bar{x}(0)>\bar{x}(\bar{u})=\bar{x}^{\bar{u}}$, the non trivial equilibria of (2.5) with $u=0$ and $u=\bar{u}<f^{\prime}(0)-r$ as control, respectively.

Note that the study of the present section is in line with (Masci et al. 2010), where productivity optimization in a constant light for a Droop model with light attenuation was considered. In that study, the analysis was much complicated by the link between 
shading and nitrogen content of the algae, so that both $S_{i n}$ and $D$ had to be taken into account; this inherent complexity prevents the direct extension of that result to the day-night setting.

\section{Productivity optimization in day/night environment}

In an environment with varying light, a non constant input must be considered. Here we consider the case where the photobioreactor is operated on the long term, with a daily biomass production from the reactor outlet. The problem that we thus consider is the maximization of the biomass production over a single day

$$
\max _{u(.) \in \mathcal{U}} \int_{0}^{T}(u(t) A) x(t) \mathrm{d} t,
$$

where $\mathcal{U}$ is the set of measurable functions such that $0 \leq u(t) \leq \bar{u} \forall t \in[0, T]$. We are then looking for a periodic regime, where the photobioreactor is operated identically each day. This means that the initial condition at the beginning of the day should equal the final condition at the end of the day. This then requires that we add the constraint

$$
x(T)=x(0) .
$$

In actual applications, the length of the bright phase will change slightly from one day to the next. This will probably impose a slight change of biomass at the beginning of the next day but, in this preliminary study, we will consider that such a phenomenon has little effect on the qualitative form of the solutions.

We therefore are faced with the following Optimal Control Problem:

$$
\begin{array}{rl}
\max _{u(t) \in[0, \bar{u}]} \int_{0}^{T} & u(t) x(t) \mathrm{d} t \\
\text { with } \quad & \dot{x}=f(x) h(t)-r x-u x, \\
& x(T)=x(0) .
\end{array}
$$

\subsection{Dealing with the T-periodicity}

In order to solve this problem, it is convenient to observe that $x(T)=x(0)=x_{0}$ cannot be achieved for all values of $x_{0}$ even without requiring optimality. For that, we first consider $u=0$ for all $t$, which yields the largest value of $x(T)$ since no biomass is taken out of the system. We first see that, for $x_{0} \geq \bar{x}^{0}$ (the equilibrium of system (2.5) with $u=0$ ), we have $x(T)<x_{0}$ because $x(t)$ is then decreasing all along the solution (or at least constant and then decreasing for $x_{0}=\bar{x}^{0}$ ). The initial condition for the solution of (4.9) must then satisfy $x_{0}<\bar{x}^{0}$. This also imposes that, in the solution of (4.9), $x(t)<\bar{x}^{0}$ for all $t$. Indeed, in the bright phase, $\dot{x} \leq 0$ at $x=\bar{x}^{0}$ (which at best allows for convergence of $x(t)$ to $\bar{x}^{0}$ in infinite time), while $\dot{x}<0$ in the dark phase.

In order to guarantee that the periodicity can be achieved for some $x_{0}>0$, we will then concentrate on what happens for $x_{0}$ in a small neighborhood of 0 . Property 1 implies that the (2.5) dynamics with $u=0$ are upper-bounded:

$$
\dot{x}<\left(f^{\prime}(0)-r\right) x,
$$


for $x>0$, so that $x(\bar{T})<x_{0} \mathrm{e}^{\left(f^{\prime}(0)-r\right) \bar{T}}$ and $x(T)=x(\bar{T}) \mathrm{e}^{-r(T-\bar{T})}<x_{0} \mathrm{e}^{\left(f^{\prime}(0)-r\right) \bar{T}} \mathrm{e}^{-r(T-\bar{T})}$. In order to have the upper-bound on $x(T)$ larger than $x_{0}$ (for all $x_{0}>0$ ), the exponential factor needs to be larger than 1, that is Assumption 1-(ii) needs to be satisfied. Considering now a small interval $(0, \epsilon]$ for the initial condition, $x(t) \leq x(\bar{T})<$ $\epsilon \mathrm{e}^{\left(f^{\prime}(0)-r\right) \bar{T}}=\epsilon_{M}$, for all $t \in[0, \bar{T}]$. We then have, from Property 1 , that $f(x)>$ $f^{\prime}(x) x>f^{\prime}\left(\epsilon_{M}\right) x$ for $x \in\left(0, \epsilon_{M}\right]$. For all $t \in[0, \bar{T}]$, we then have $\dot{x}>\left(f^{\prime}\left(\epsilon_{M}\right)-\right.$ $r) x$ and $x(\bar{T})>x_{0} \mathrm{e}^{\left(f^{\prime}\left(\epsilon_{M}\right)-r\right) \bar{T}}$, so that $x(T)>x_{0} e^{\left(f^{\prime}\left(\epsilon_{M}\right) \bar{T}-r T\right)}$. In order to have $x(T)>x_{0}$, it then suffices to have $f^{\prime}\left(\epsilon_{M}\right) \bar{T}-r T>0$ for some $\epsilon>0$. Provided Assumption 1-(ii) is satisfied, it suffices to take $\epsilon$ small enough, and $x(T)>x_{0}$ for all $x_{0} \in(0, \epsilon]$. Assumption (1)-(ii) is therefore both necessary and sufficient to ensure that there exists some $x_{0}$ that has $x(T)>x_{0}$ with $u(t)=0$ for all $t$.

We see there the importance of this assumption, which is quite natural since it imposes that, when the population is small, that is when the growth rate is the largest, growth during the daylight period exceeds the net effect of respiration that takes place during the whole day.

\subsection{Maximum principle}

In this section, we will show that the solution of (4.9) can have one of three patterns. In order to solve problem (4.9), one option would be to use the maximum principle for hybrid systems (Sussmann 1999) but, given the simplicity of our setting, we will simply use the original Pontryagin's Maximum Principle (PMP, Pontryagin, Boltyansky, Gamkrelidze \& Mishchenko (1962)) in looking for a control law maximizing the Hamiltonian

$$
H(x, u, \lambda, t) \triangleq \lambda(f(x) h(t)-r x-u x)+u x,
$$

with the constraint

$$
\left\{\begin{array}{l}
\dot{x}=f(x) h(t)-r x-u x \\
\dot{\lambda}=\lambda\left(-f^{\prime}(x) h(t)+r+u\right)-u
\end{array}\right.
$$

In addition, we should add the constraint

$$
\lambda(T)=\lambda(0) .
$$

as shown in (Gilbert 1977). We see from the form of the Hamiltonian that

$$
\frac{\partial H}{\partial u}=(1-\lambda) x
$$

so that, when $\lambda>1$, we have $u=0$, when $\lambda<1$, we have $u=\bar{u}$, and when $\lambda=1$ over some time interval, intermediate singular control might be applied. Note that, despite the discontinuity in time, no jump in the adjoint variable occurs; such a jump would only occur if using the Hamiltonian for the system extended with time as a second state, and would only concern the corresponding second adjoint state. Due to the simplicity of the time-dependence, the use of the extended system was not needed.

Of paramount importance in the proofs will be the constancy of the Hamiltonian. Indeed, it is known that the Hamiltonian is constant along optimal solutions as long as the time does not intervene into the dynamics or the payoff. Since this constancy is rooted in infinitesimal computations (in regions where $u(t)$ is constant, it suffices to check $\dot{H})$, this constancy is preserved in the intervals $[0, \bar{T})$ and $(\bar{T}, T]$. The Hamilto- 
nian presents a discontinuity at $\bar{T}$, which would not have occurred if using the Hamiltonian for the system with time as a second state. The adjoint variables, however, remain continuous in time at that point through the maximum principle, though their derivatives can present a discontinuity.

We can first give some general statements about where and when switches can occur

Proposition 1 If Assumption 1 is satisfied then, a solution of problem (4.9) satisfies the following properties

(i) No switch from $u=0$ to $u=\bar{u}$ can take place in the dark phase;

(ii) Switches from $u=0$ to $u=\bar{u}$ in the bright phase take place with $x \leq x_{\sigma}$;

(iii) Switches from $u=\bar{u}$ to $u=0$ in the bright phase take place with $x \geq x_{\sigma}$.

Proof: All these results come from the analysis of $\dot{\lambda}$ at the switching instant. Indeed, at a switching instant, we have $\lambda=1$ so that:

$$
\dot{\lambda}=\lambda\left(-f^{\prime}(x) h(t)+r\right) \text {. }
$$

The form of $\frac{\partial H}{\partial u}$ indicates that a switch from $u=0$ to $u=\bar{u}$ (resp. from $u=\bar{u}$ to $u=0$ ) can only occur if $\dot{\lambda} \leq 0$ (resp. $\dot{\lambda} \geq 0$ ).

In the dark phase, (4.11) becomes $\dot{\lambda}=r \lambda>0$; no switch from $u=0$ to $u=\bar{u}$ can therefore take place (i).

In the bright phase, $\dot{\lambda} \leq 0$ at a switching instant if $f^{\prime}(x) \geq r$, which is equivalent to having $x \leq x_{\sigma}$; this is therefore a condition for a switch from $u=0$ to $u=\bar{u}$ (this shows (ii)). Conversely for (iii).

Point (i) is in fact quite natural: if a bioreactor was closed at some moment of the night, opening it later during the night would mean that some biomass would have been uselessly respirated before that. The following theorem gives all possible solutions of the PMP

Theorem 1 If Assumption 1 is satisfied then three forms of control are possible in solutions of problem (4.9):

(a) Bang-bang with $u(0)=0$, a single switch to $u(t)=\bar{u}$ taking place strictly before $\bar{T}$ and a single switch back to $u(t)=0$ taking place strictly after $\bar{T}$;

(b) Bang-singular-bang with $u(0)=0$, a switch to $u(t)=u_{\sigma}$ taking place first, a single switch to $u(t)=\bar{u}$ taking place strictly before $\bar{T}$ and a single switch back to $u(t)=0$ taking place strictly after $\bar{T}$.

(c) Constant control at $u(t)=\bar{u}$

In the first two cases, the switch back to $u(t)=0$ takes place with $x$ strictly smaller than it was at the moment of switch to $u(t)=\bar{u}$. 
Without needing an explicit form of $f(x)$, we have been able to obtain the qualitative form of the optimal solution analytically. The proof of this result is detailed in appendix and is obtained by considering all possible situations that are in concordance with Proposition 1 and eventually eliminating all possibilities but the three cases detailed in Theorem 1. The link with the optimal solution for the day-night version of model (2.3) is then completed by imposing a very high $S_{i n}$ during the open-reactor phases so that the substrate density is kept high and $\mu_{S}(S) \approx \bar{\mu}$ at all times.

In the bang-bang case, the optimal solution is made of four phases: (i) growth with a closed photobioreactor until a sufficient biomass level is reached - (ii) maximal harvesting with simultaneous growth - (iii) maximal harvesting, without growth, of the biomass produced during the light phase while not going below the level where the residual biomass left is sufficient to efficiently start again the next day - (iv) passive bioreactor (no harvesting, no growth, only respiration).

In the bang-singular-bang case, a (i-bis) singular phase is added between (i) and (ii) compared with the bang-bang case: during this whole phase, maximal instantaneous productivity is achieved. This solution with a singular form then seems to be naturally the most efficient one. It can however not always be achieved for two reasons:

- if $u_{\sigma}>\bar{u}, u_{\sigma}$ is not an admissible control. The phase where $u=\bar{u}$ in the bangbang case then parallels the singular phase since, in both cases, they correspond to instantaneous productivity optimization.

- if growth is not sufficiently stronger than respiration (with Assumption 1-(ii) satisfied however), there might not be enough time for $x$ to reach $x_{\sigma}$ during the light phase.

The only remaining solution is the one with $u$ constant at $\bar{u}$ and is characterized by constant maximal harvesting when the actuator has been under-dimensioned and when the dark phase does not last too long, otherwise the respiration and/or the maximal dilution would wash-out the reactor. Finally, note that the phases where the reactor is closed at the beginning of the day is there to allow for the biomass to go back up to the level where productivity is high, so that, when the night duration goes to zero, the phases where $u=0$ disappear. A similar reasoning can be held for the $u=\bar{u}$ phase in case (b), which harvests the biomass at maximal rate to limit respiration at night. There is thus clear continuity between these day-night solutions as the night length goes to zero and the constant light optimal ones.

\section{Discussion}

\subsection{Existence and stability of the optimal solution}

Existence of an optimal solution is obvious since the achieved yield for solutions satisfying $x(T)=x(0)$ is bounded between 0 (obtained for $u=0$ ) and $\bar{x}^{0} \bar{u} A T$ (not achievable but an upper-bound nonetheless because $x(t) \leq \bar{x}^{0}$ and $u(t) \leq \bar{u}$ at all times). The productivity level therefore has a finite supremum, which translates into a maximum since the yield is continuous in $u(t)$ and the set of definition of the $u(t)$ control laws bounded by $[0, \bar{u}]$ is compact. The optimal control problem therefore has a solution. Note that if $f(x)$ was linear, no solution of the optimal control problem 
would exist because, for a given $u(t)$ that ensures $x(T)=x(0)$, linearity yields that this is achieved for any $x(0)$ with a payoff linear in $x(0)$. Optimality then requires $x(0) \rightarrow+\infty$.

Set in a periodic framework, where it would repeat itself over time, we will show that the optimal solution is also asymptotically stable. Indeed, for given periodic $u^{*}(t)$ and $x^{*}(t)$ (defined for $t \in \mathbb{R}_{+}$) built on an optimal control law of Theorem 1 and the corresponding optimal solution, the solution of the periodic use of equation (2.7), with $u=u^{*}(t)$ and some $x(0)>0$, generates a solution $x(t)$ (defined for $t \in \mathbb{R}_{+}$). Convergence of $x(t)$ to $x^{*}(t)$ is then shown by proving that the $(x(n T))_{n \in \mathbb{N}}$ sequence is converging to $x^{*}(0)>0$. For that, we consider $x(0) \neq x^{*}(0)$ (which implies $x(t) \neq x^{*}(t)$ at all times through uniqueness of solutions considerations). Building now the function

$$
W\left(x(t), x^{*}(t)\right)=\frac{1}{2}\left(\ln (x(t))-\ln \left(x^{*}(t)\right)\right)^{2} \geq 0,
$$

its time derivative along $(2.7)$ is

$$
\dot{W}=h(t)\left(\frac{f(x(t))}{x(t)}-\frac{f\left(x^{*}(t)\right)}{x^{*}(t)}\right)\left(\ln (x(t))-\ln \left(x^{*}(t)\right)\right)
$$

which is zero in the dark phase and negative in the light phase because $\frac{f(x)}{x}$ is a decreasing function while $\ln (x)$ is an increasing function of $x$. We conclude that $W\left(x((n+1) T), x^{*}((n+1) T)\right)<W\left(x(n T), x^{*}(n T)\right)$ which, since $x^{*}$ is $T$-periodic. is equivalent to

$W\left(x((n+1) T), x^{*}(0)\right)<W\left(x(n T), x^{*}(0)\right)$. We then have that $\left(W\left(x(n T), x^{*}(0)\right)\right)_{n \in \mathbb{N}} \geq$ 0 is a decreasing, hence converging, sequence. The asymptotic behavior of $(x(n T))_{n \in \mathbb{N}}$ is then characterized by having $\left(W\left(x(n T), x^{*}(0)\right)\right)_{n \in \mathbb{N}}$ constant. Since constancy requires to have $x(t)=x^{*}(t)$ in the whole light phase to keep $\dot{W}=0,(x(n T))_{n \in \mathbb{N}}$ necessarily converges to $x^{*}(0)$, so that the periodic $x^{*}(t)$ is also globally asymptotically stable.

This property is quite useful since it guarantees that, when applying the periodic optimal control over several days, the biomass level will automatically be steered to the level that ensures optimal yield.

\subsection{Practical implementation}

The computation of the solution of the optimal control problem (4.9) gives us two things: $u^{*}(t)(t \in[0, T])$, the optimal control, and $x^{*}(t)(t \in[0, T])$ the biomass level evolution. The latter fixes the initial and final condition $x^{*}(0)=x^{*}(T)$. Once this is set, two points are important during the implementation on a real plant: the final condition should be the desired $x^{*}(T)$ so that production can start again the next day and the biomass productivity should be maximized. In the absence of perturbation and with the correct $x(0)=x^{*}(0)$, this is easily achieved through $u^{*}(t)$. If the system suffers from disturbances, inaccurate initial conditions or presents some unmodelled dynamics, neither will be achieved. With the hypothesis that the previously computed $x^{*}(T)$ is desirable despite these perturbations, a simple solution to limit their consequences would be to set our problem in a setting close to a Model Predictive Control framework (Rawlings 2000), but without receding horizon: the time interval $[0, T]$ is divided into $m$ (for some fixed $m \in \mathbb{N}$ ) smaller intervals of length $\frac{T}{m}$ at the beginning of which 
the solution of the problem

$$
\begin{aligned}
& \max _{u(t) \in[0, \bar{u}]} \int_{\frac{j T}{m}}^{T}(u(t) A) x(t) \mathrm{d} t \\
& \text { with } x\left(\frac{j T}{m}\right) \text { given and } x(T)=x^{*}(T)
\end{aligned}
$$

is computed, for $j \in\{0, \cdots, m-1\}$ and with the $x$ dynamics given in (2.7). In detail, once a control (computed by the present MPC-like scheme) has been applied up to time $\frac{j T}{m}$ on the real plant, the biomass level $x\left(\frac{j T}{m}\right)$ is measured and problem (5.12) is either solved online through an efficient algorithm (e.g. shooting method (Betts 2010)) or uses the pre-computed solution of (5.12) that would have been achieved offline for all initial conditions. The obtained control $u(t)$ is then applied between times $x\left(\frac{j T}{m}\right)$ and $x\left(\frac{(j+1) T}{m}\right)$, where the procedure is started again. Note that the solution of (5.12) is linked to the value function of the original problem since it gives the cost-to-go at time $\frac{j T}{m}$; this is a link between this scheme and a construction of an explicit feedback built on the Hamilton-Jacobi-Bellman equation, which hinges on the explicit construction of the value function.

Note that, due to perturbations having occured or unmodelled dynamics, there might be some $j$ for which problem (5.12) has no solution because the terminal constraint $x(T)=x^{*}(T)$ cannot be satisfied for any control. In that case, the productivity objective is dropped and problem (5.12) is replaced by $\min _{u(t) \in[0, \bar{u}]}\left|x(T)-x^{*}(T)\right|$ for the given $x\left(\frac{j T}{m}\right)$. Monotonicity of $x(T)$ with respect to $u(t)$ implies that the solution of this problem is trivially $u(t)=\bar{u}$ (resp. $u=0$ ) if the constraint $x(T)=x^{*}(T)$ in (5.12) cannot be satisfied because $x(T)>x^{*}(T)$ (resp. $x(T)<x^{*}(T)$ ) independently of $u(t)$. The procedure is then started again at time $\frac{(j+1) T}{m}$ by first evaluating if (5.12) has a solution. This strategy is chosen because the terminal constraint is deemed more important than the yield for the perenity of the process.

\section{Simulations}

In this section, we concentrate on model (2.4), which means that the high substrate level hypothesis is supposed to be valid through a high value of $S_{i n}$ in model (2.3). Simulations of the solution of (4.9) are then performed with $f(x)=\frac{\bar{\mu}}{a} \ln \frac{\bar{I}_{0}+K_{I}}{\bar{I}_{0} \mathrm{e}^{-a x}+K_{I}}$.

\subsection{Results for the microalgae Isochrysis galbana}

We will now analyze the forms of solutions (a), (b), and (c) of Theorem 1. For that, we start with a dynamical model (2.4) for the growth of Isochrysis galbana with the parameters taken as in (Masci et al. 2010).

With such parameters, the optimal solution, represented by the blue curves in Figure 1 is of the bang-singular-bang type and achieves a daily surfacic productivity of $6.33 g[C] / m^{2}$ for a total cumulated flow $\int_{0}^{1} u(\tau) \mathrm{d} \tau$ equal to 0.453 , that is $45 \%$ of the medium has been renewed during the 24 hours. We then considered the application of a constant control during the 24 hours and optimized the level of this control numerically. The optimum was achieved for $\hat{u}=0.461$, which yields a daily productivity 


\begin{tabular}{|c|c|c|}
\hline Parameter & Value & Units \\
\hline \hline $\bar{\mu}$ & 1.7 & $d a y^{-1}$ \\
$a$ & 0.5 & $m^{2} / g[C]$ \\
$\bar{I}_{0}$ & 1500 & $\mu$ mol quanta $m^{-2} s^{-1}$ \\
$K_{I}$ & 20 & $\mu$ mol quanta $m^{-2} s^{-1}$ \\
$r$ & 0.07 & $d a y^{-1}$ \\
$\bar{T}$ & 0.5 & $d a y$ \\
$\bar{u}$ & 2 & $d a y^{-1}$ \\
\hline
\end{tabular}

Table 1: Growth and bioreactor parameters for Isochrysis galbana

equal to $6.26 \mathrm{~g}[\mathrm{C}] / \mathrm{m}^{2}$ and a cumulated flow equal to 0,461 also. Two points need to be noticed from this comparison: (i) the productivity improvement with the optimal solution, compared to the best constant control, is very weak (1.11\%); (ii) the total flows required to attain both optimal solutions are very similar. The fact that the improvement of the productivity is small is not surprising: the necessity of shutting down the chemostat at night is linked to the respiration that would consume the biomass. In the present case, the respiration is weak so that, during one night, only $3.44 \%$ of the biomass is consumed. This phenomenon is here marginal so that the optimal control approach developed to limit it provides little gain and what really matters is the total flow that goes through the photobioreactor. Almost any control that achieves large values of the biomass will yield near-optimal productivities because this large biomass is not penalized by respiration.

In order to cover all cases of Theorem 1, we considered two other situations: $\bar{u}=$ 0.8 and $\bar{u}=0.1$, which prevent the existence of a singular phase because $\bar{u}<u_{\sigma}$. For $\bar{u}=0.8$, the optimal solution is bang-bang with a productivity level very close to the previous case $\left(6.30 \mathrm{~g}[\mathrm{C}] / \mathrm{m}^{2}\right)$ and the total flow also varies little (0.457). With $\bar{u}=0.1$, a total flow close to 0.46 cannot be achieved and a significant performance degradation occurs: $u(t)$ is constant at $u=0.1$ and the productivity drops to $4.35 g[C] / \mathrm{m}^{2}$.

\subsection{Importance of the respiration}

In this section we explore the impact of a larger value for parameter $r$, which can be due to increased respiration, or to a high mortality as often is the case in high density cultures (however, we will stick to the respiration terminology). If we now consider Isochrysis galbana in an environment where mortality brings $r$ to $0.7 d a y^{-1}$, we expect the optimal strategy to have a much bigger impact on the outcome. Indeed, such a respiration level has much more importance at night than in the previous case since it consumes $29.5 \%$ of the biomass, hence the importance of limiting the biomass level at night. We see in Figure 2 that the optimal solution is here bang-bang with a short opening window at the end of the day and at the beginning of the night to harvest the produced biomass $(u=\bar{u}$ for $t \in[0.479,0.527])$. The optimal production is here $0.607 \mathrm{~g}[\mathrm{C}] / \mathrm{m}^{2}$ for a total flow of 0.096 , that is a very little daily medium renewal while the best constant control $\hat{u}=0.095$ yields $0.519 g[C] / m^{2}$. We see here that the daily total flows are again almost equivalent but that, because respiration is limited by the early harvest, the productivity is here improved by $17 \%$ through the bang-bang approach, which is far from being negligible, especially since it is made with almost 

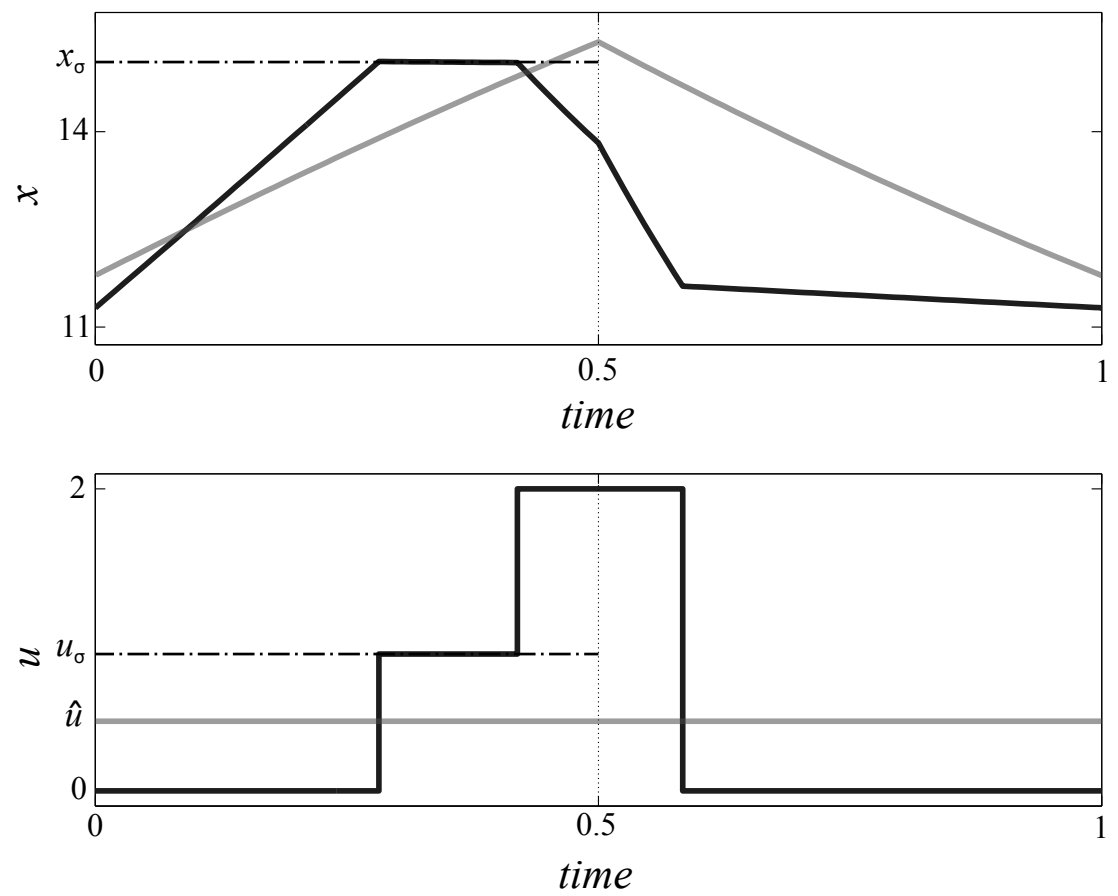

Figure 1: Bang-singular-bang optimal solution (in black) for model (2.4) confronted with the most productive constant dilution rate scenario (in grey) for the microalgae Isochrysis galbana and the parameters of Table 1. At the top is the evolution of the biomass and at the bottom that of the control. The black dash-dotted lines represent the values of $x_{\sigma}$ and $u_{\sigma}$ respectively

the same cumulated through-flow, hence consumed energy, as the best constant control case.

\subsection{Near-optimal strategies}

We have seen that the daily flow that goes through the chemostat has great importance for the productivity level of a solution: in the small respiration case, the bang-bang controls with $\bar{u}=2$ or $\bar{u}=0.8$ and the constant control with $\hat{u}=0.461$ have almost the same productivity, while having very similar total flow going through the reactor. In order to exploit that property that, we propose a strategy that is of the bang-bang type and consists in having the dilution equal to $\bar{u}$ in the interval $\left[\bar{T}-\frac{\tilde{u}}{2 \bar{u}}, \bar{T}+\frac{\tilde{u}}{2 \bar{u}}\right]$ and 0 outside of this interval. That way, the total flow that goes through the reactor is equal to $\tilde{u}$, so that we will be able to compare the obtained productivity level between that strategy and constant control strategies that have the same daily total flow. Being bang-bang, this strategy has the form of an optimal solution defined in Theorem 1; however, the switching times are most probably not the ones that the PMP would prescribe, so that only near-optimality is achieved. We did the computations for $\bar{u}=2$ and values of $\tilde{u}$ that did not lead to the wash-out of the reactor for species of subsection 6.2 (Figure 3). We see that, the constant control at value $\tilde{u}$ (in red) yields a less productive process than 

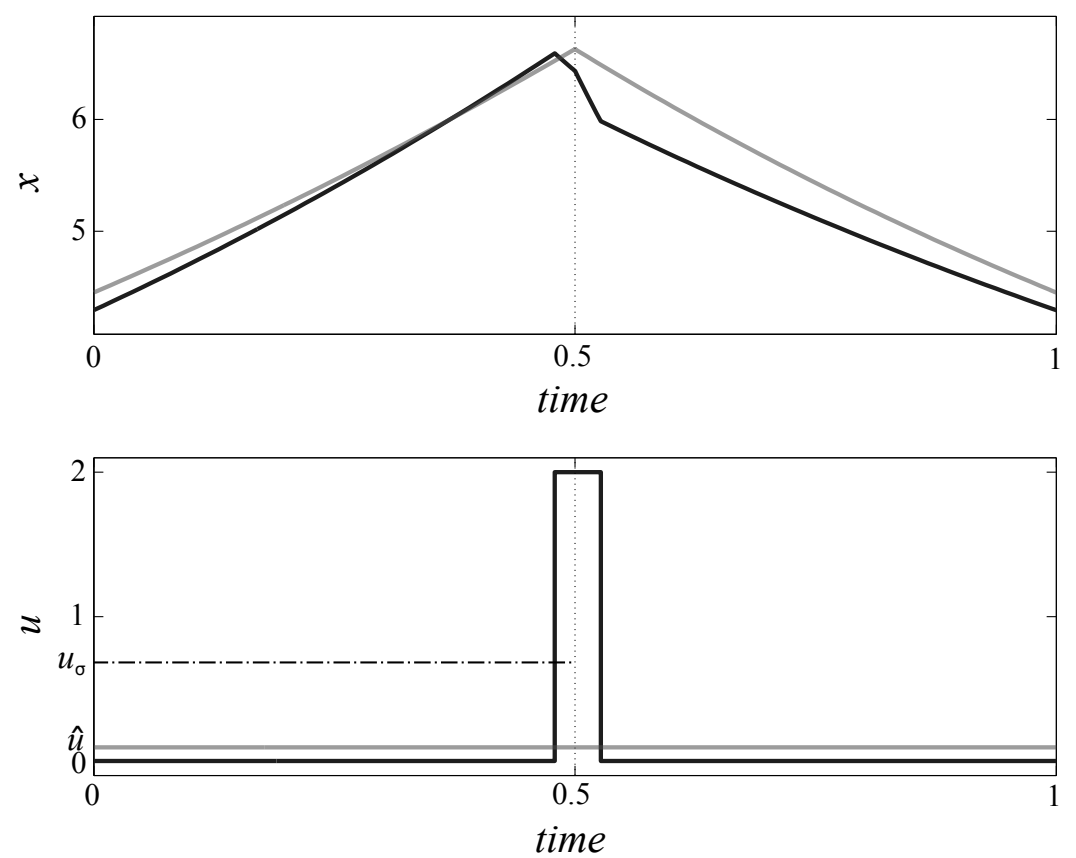

Figure 2: Bang-bang optimal solution (in black) for model (2.4) confronted with the most productive constant dilution rate scenario (in grey) for a high-respiration species $(r=0.7)$ and $\bar{u}=2$

the corresponding suboptimal bang-bang strategy. Therefore, when the exact optimal control law is not computed, it is advantageous to choose a bang-bang one rather than a constant control. This strategy is strongly advisable since the optimal productivity level is represented through a dotted level in the figure and the near-optimal strategy achieves it almost for an appropriate daily flow. This was expected in the high respiration case where the optimal solution is bang-bang, as would also be the case in a low-respiration case where the optimal solution is bang-singular-bang, where respiration is not too detrimental if $x(t)$ goes above $x_{\sigma}$. Such would probably not be the case when confronting the near-optimal strategy to a bang-singular-bang optimal pattern that would occur in a high respiration case.

\section{Conclusions}

In this paper, we have shown how the day-night constraint influences the optimal control strategy that achieves maximal biomass daily productivity in a photobioreactor and reduces the optimal productivity level. We have identified three families of strategies that can achieve optimality: bang-singular-bang, bang-bang, and constant maximal control; the first two are characterized by an harvesting of the biomass at the end of the light phase and beginning of the night to limit the negative effects of the respiration, while the latter leads to permanent harvesting because the maximal dilution rate is too weak compared with the growth rate of the biomass. These families of control 


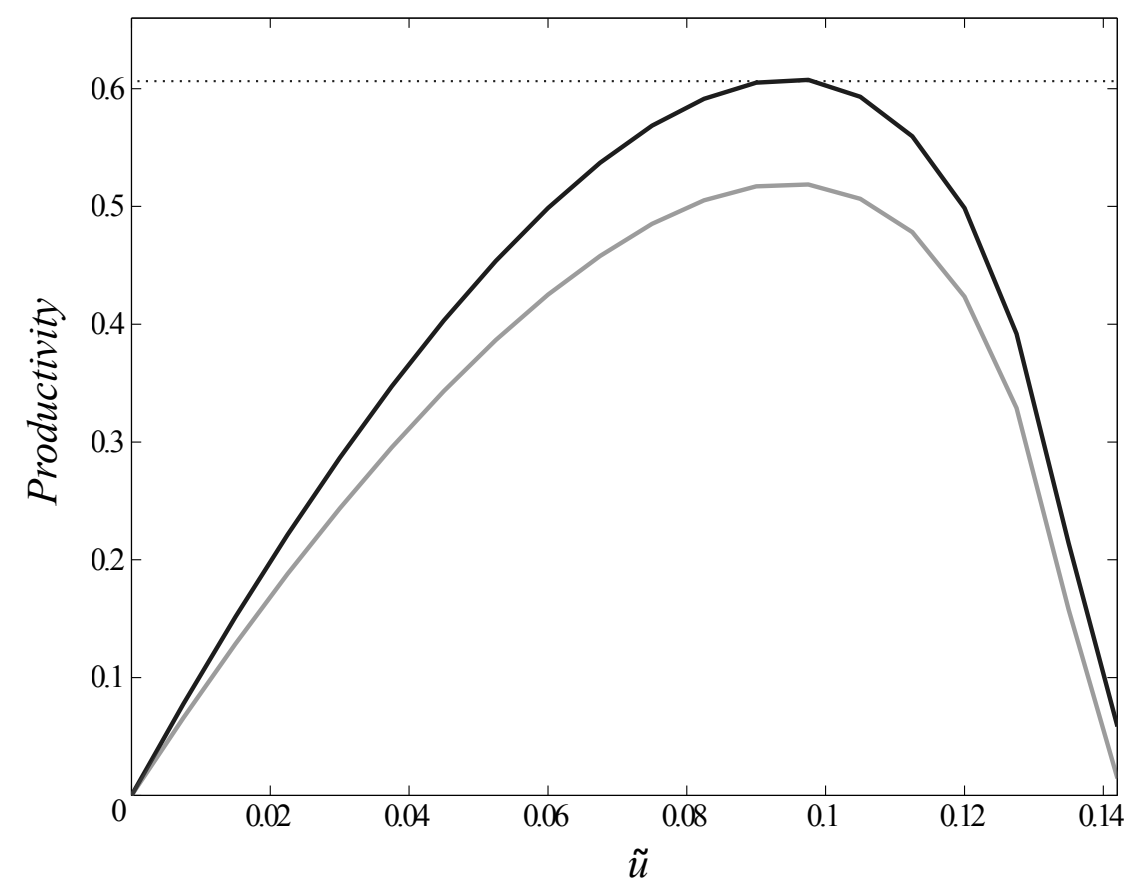

Figure 3: Productivity levels of species of Section 6.2: in grey, the productivity attained with the constant control $u=\tilde{u}$ and in black the one with the near-optimal strategy of Section 6.3. The dashed-line represent the optimal productivity levels with $\bar{u}=2$

strategies have been built for a large set of nonlinear one-dimensional models with light-dark phases so that they can be applied beyond their motivation in this paper: that is a photobioreactor with Monod-like growth and Beer-Lambert light attenuation. Such a formalism even allows to go beyond chemostat applications and consider populations that grow in more general periodic environments (see Grognard, Akhmetzhanov \& Bernard (2012) for an example).

Through simulations, we have shown that the necessity of applying an optimal control strategy strongly depends on the respiration rate: if the latter is weak, constant control can achieve almost the same performance since the night phase consumes little biomass. However, we have also shown that a better choice is probably to apply a bangbang control law which, in the presented simulations, always yields better productivity than comparable strategies with constant dilution. This is particularly supported by the fact this better productivity is achieved with a very similar cumulated effort as the one necessary for a constant dilution rate.

\section{Acknowledgments}

We are very grateful to the three reviewers who carefully read the manuscript and strongly contributed to its improvement. 


\section{References}

Becerra-Celis, G., Hafidi, G., Tebbani, S., Dumur, D. \& Isambert, A. (2008). Nonlinear predictive control for continuous microalgae cultivation process in a photobioreactor, Control, Automation, Robotics and Vision, 2008. ICARCV 2008. 10th International Conference on, pp. $1373-1378$.

Bernard, O. (2011). Hurdles and challenges for modelling and control of microalgae for CO2 mitigation and biofuel production, Journal of Process Control 21(10): 13781389.

Bernard, O., Masci, P. \& Sciandra, A. (2009). A photobioreactor model in nitrogen limited conditions, 6th Vienna International Conference on Mathematical Modelling MATHMOD 2009, Vienna, Austria.

Betts, J. (2010). Practical Methods for Optimal Control and Estimation Using Nonlinear Programming, Advances in design and control, Society for Industrial and Applied Mathematics (SIAM, 3600 Market Street, Floor 6, Philadelphia, PA 19104).

URL: http://books.google.fr/books?id=n9hLriD8Lb8C

Chisti, Y. (2007). Biodisel from microalgae, Biotechnology Advances 25: 294-306.

Clark, C. (1990). Mathematical Bioeconomics: The Optimal Management of Renewable Resources, Wiley Interscience.

Dong, L., Chen, L. \& Sun, L. (2007). Optimal harvesting policies for periodic gompertz systems, Nonlinear Analysis: Real World Applications 8(2): 572 - 578.

Droop, M. (1968). Vitamin b12 and marine ecology. iv. the kinetics of uptake growth and inhibition in monochrysis lutheri, J. Mar. Biol. Assoc. 48(3): 689-733.

Gilbert, E. G. (1977). Optimal periodic control: A general theory of necessary conditions, SIAM Journal on Control and Optimization 15(5): 717-746.

Grobbelaar, J. \& Soeder, C. (1985). Respiration losses in planktonic green algae cultivated in raceway ponds, J. Plankton Research 7(4): 497-506.

Grognard, F., Akhmetzhanov, A., Masci, P. \& Bernard, O. (2010). Optimization of a photobioreactor biomass production using natural light, Proceedings of the 49th IEEE CDC conference, Atlanta, USA.

Grognard, F., Akhmetzhanov, R., A. \& Bernard, O. (2012). Periodic optimal control for biomass productivity maximization in a photobioreactor using natural light, Rapport de recherche RR-7929, INRIA.

URL: http://hal.inria.fr/hal-00687322

Guardabassi, G., Locatelli, A. \& Rinaldi, S. (1974). Status of periodic optimization of dynamical systems, Journal of Optimization Theory and Applications 14: 1-20. 10.1007/BF00933171.

Hoffmann, J. (1998). Wastewater treatment with suspended and non-suspended algae, J. Phycol. 34: 757-763. 
Huisman, J., Matthijs, H., Visser, P., Balke, H., Sigon, C., Passarge, J., Weissing, F. \& Mur, L. (2002). Principles of the light-limited chemostat: theory and ecological applications, Antonie van Leeuwenhoek 81: 117-133. 10.1023/A:1020537928216.

Huntley, M. \& Redalje, D. (2007). CO2 mitigation and renewable oil from photosynthetic microbes: A new appraisal, Mitigation and Adaptation Strategies for Global Change 12(4): 573-608.

Kelley, H. J., Kopp, R. E. \& Gardner Moyer, H. (1967). Singular extremals, Topics in Optimization 31: 63-101.

Lambert, J. (1760). Photometria sive de mensura et gradibus luminis, colorum et umbrae, Eberhardt Klett, Germany.

Marxen, K., Vanselow, K., Lippemeier, S., Hintze, R., Ruser, A. \& Hansen, U.-P. (2005). A photobioreactor system for computer controlled cultivation of microalgae, Journal of Applied Phycology 17: 535-549. 10.1007/s10811-005-9004-8.

Masci, P., Bernard, O. \& Grognard, F. (2010). Microalgal biomass productivity optimization based on a photobioreactor model, Proceedings of the 11th Computer Applications in Biotechnology Symposium, Leuven, Belgium.

Monod, J. (1942). Recherches sur la Croissance des Cultures Bactériennes, Herman, Paris.

Oswald, W. (1988). Micro-algal Biotechnology, Cambridge University Press, chapter Micro-algae and waste-water treatment, pp. 305-328.

Pontryagin, L., Boltyansky, V., Gamkrelidze, R. \& Mishchenko, E. (1962). Mathematical Theory of Optimal Processes, Wiley-Interscience, New York.

Rawlings, J. (2000). Tutorial overview of model predictive control, IEEE CONTROL SYSTEMS MAGAZINE 20(3): 38-52.

Smets, I. Y., Claes, J. E., November, E. J., Bastin, G. P. \& Van Impe, J. F. (2004). Optimal adaptive control of (bio)chemical reactors: past, present and future, Journal of Process Control 14(7): 795-805.

Spolaore, P., Joannis-Cassan, C., Duran, E. \& Isambert, A. (2006). Commercial applications of microalgae, Journal of Bioscience and Bioengineering 101(2): 87-96.

Suh, I. \& Lee, C.-G. (2003). Photobioreactor engineering: Design and performance, Biotechnology and Bioprocess Engineering 8: 313-321. 10.1007/BF02949274.

Sussmann, H. (1999). A maximum principle for hybrid optimal control problems, Decision and Control, 1999. Proceedings of the 38th IEEE Conference on, Vol. 1, pp. $425-430$ vol. 1 .

Tartakovsky, B., Ulitzur, S. \& Sheintuch, M. (1995). Optimal control of fed-batch fermentation with autoinduction of metabolite production, Biotechnology Progress 11(1): 80-87.

Wang, F. S. \& Shyu, C. H. (1997). Optimal feed policy for fed-batch fermentation of ethanol production by \&lt;small\&gt;zymomous mobilis\&lt;/small\&gt; Bioprocess and Biosystems Engineering 17: 63-68. 10.1007/PL00008957. 
Wu, W. T., Chen, K. C. \& Chiou, H. W. (1985). On-line optimal control for fedbatch culture of bakers yeast production., Biotechnology and Bioengineering 27(5): 756-760.

\section{A Proof of Theorem 1}

We will detail the different forms of solutions and show that no other solution than the one exposed in Theorem 1 can occur, based on a discussion on $\lambda_{0} \triangleq \lambda(0)$.

Bang-bang with $\lambda_{0}>1$ : With $\lambda_{0}>1$, we have $u=0$ at times 0 and $T$. At least, a switch of $u$ from 0 to $\bar{u}$ and back to 0 then needs to occur between time 0 and $T$; otherwise, $u(t)=0$ at all times and the payoff $\int_{0}^{T}(u(t) A) x(t) \mathrm{d} t$ would be 0 , since the argument of the integral that defines the productivity would always be 0 . Since the productivity is non-negative by constructive, bang-bang solutions that switch to $\bar{u}$ can do no worse than that and are worth examining. As we have seen in Proposition 1(i), the switch from 0 to $\bar{u}$ cannot take place in the dark phase. For the solution that we study, a switch then needs to take place at time $t_{0 \bar{u}}$ in the $(0, \bar{T})$ interval and for $x\left(t_{0 \bar{u}}\right)=x_{0 \bar{u}}<x_{\sigma}$ and $\lambda\left(t_{0 \bar{u}}\right)=1$ (Proposition 1-(ii)).

The switch from $u=\bar{u}$ to $u=0$ then needs to occur at some time $t_{\bar{u} 0}>t_{0 \bar{u}}$ (and some value of $\left.x\left(t_{\bar{u} 0}\right)=x_{\bar{u} 0}\right)$. Two possibilities then need to be considered:

- $t_{\bar{u} 0} \leq \bar{T}$ : from Proposition 1, we can deduce that $x_{\bar{u} 0} \geq x_{\sigma}$. Applying $u=0$ then forces convergence toward $\bar{x}^{0}$ for $t_{\bar{u} 0} \leq t \leq \bar{T}$, so that $x(t)$ increases, and so stays above $x_{\sigma}$ (so that $x(\bar{T}) \triangleq x_{\bar{T}} \geq x_{\sigma}>x_{0}$ ). No switch back to $u=\bar{u}$ can then take place neither before $\bar{T}$ (because of (ii) of Proposition 1) nor after $\bar{T}$ (because of (i) of Proposition 1).

- $t_{\bar{u} 0}>\bar{T}$ : from Proposition 1-(i), we can deduce that no other switch takes place afterward. Two cases will then be considered: either $x_{0 \bar{u}} \geq \bar{x}^{\bar{u}}$ so that $x(t)$ decreases and $x_{0 \bar{u}} \geq x_{\bar{T}}>x_{\bar{u} 0}$ or $x_{0 \bar{u}}<\bar{x}^{\bar{u}}$ so that $x_{0 \bar{u}}<x_{\bar{T}}$, and we do not necessarily know if $x_{0 \bar{u}}>x_{\bar{u} 0}$

We have then shown that any bang-bang solution with $u(0)=0$ presents a single switch to $u=\bar{u}$ (before $\bar{T}$ ) and a single switch to $u=0$ (before or after $\bar{T}$ ). We are then left with two things to show: first that $t_{\bar{u} 0} \leq \bar{T}$ leads to a contradiction and then that, when $x_{0 \bar{u}}<\bar{x}^{\bar{u}}$, we indeed have $x_{0 \bar{u}}>x_{\bar{u} 0}$.

We will now show that the switch from $\bar{u}$ to 0 needs to occur strictly after $\bar{T}$. For that, we will consider that $t_{\bar{u} 0} \leq \bar{T}$ and show that this leads to a contradiction. Using the constancy of the Hamiltonian in $[0, \bar{T})$, we have, by continuity of the variables at time $\bar{T}$, that

$$
\lambda_{0}\left(f\left(x_{0}\right)-r x_{0}\right)=\lambda_{\bar{T}}\left(f\left(x_{\bar{T}}\right)-r x_{\bar{T}}\right),
$$

and, in $(\bar{T}, T]$, with $x(T)=x_{0}$ and $\lambda(T)=\lambda_{0}$ :

$$
\lambda_{0}\left(-r x_{0}\right)=\lambda_{\bar{T}}\left(-r x_{\bar{T}}\right) .
$$

Taking the differences between the equalities, we obtain

$$
\lambda_{0} f\left(x_{0}\right)=\lambda_{\bar{T}} f\left(x_{\bar{T}}\right) .
$$


Taking the quotient of the last two equalities then yields

$$
\frac{f\left(x_{0}\right)}{x_{0}}=\frac{f\left(x_{\bar{T}}\right)}{x_{\bar{T}}},
$$

Remembering from Property 1 that $\frac{f(x)}{x}$ is a strictly decreasing function, this leads to a contradiction because $x_{\bar{T}}>x_{0}$ since the biomass only decreases at night. The switch from $u=\bar{u}$ to $u=0$ then takes place with $t_{\bar{u} 0}>\bar{T}$.

We will now show that, even in the case where $x_{0 \bar{u}}<\bar{x}^{\bar{u}}$, the second switch takes place with $x_{\bar{u} 0}<x_{0 \bar{u}}$. For that, we first note that, since no switch from $u=\bar{u}$ to $u=0$ takes place in the $\left[t_{0 \bar{u}}, \bar{T}\right]$ interval, $f\left(x_{\bar{T}}\right)-r x_{\bar{T}}>f\left(x_{0 \bar{u}}\right)-r x_{0 \bar{u}}$. Indeed, since $x(t)$ is increasing along the considered solutions from $x_{0 \bar{u}}<x_{\sigma}, f(x(t))-r x(t)$ first increases from $f\left(x_{0 \bar{u}}\right)-r x_{0 \bar{u}}$ and then decreases (once $x(t)$ gets above $x_{\sigma}$ ); if we had $f\left(x_{\bar{T}}\right)-r x_{\bar{T}} \leq f\left(x_{0 \bar{u}}\right)-r x_{0 \bar{u}}$, there would be a time $\tilde{t}$ belonging to the interval $\left(t_{0 \bar{u}}, \bar{T}\right]$ where $f(x(\tilde{t}))-r x(\tilde{t})=f\left(x_{0 \bar{u}}\right)-r x_{0 \bar{u}}$. A switch then necessarily would have taken place at that instant because of constancy of the Hamiltonian

$$
\left(f\left(x_{0 \bar{u}}\right)-r x_{0 \bar{u}}\right)=\lambda(\tilde{t})(f(x(\tilde{t}))-(r+\bar{u}) x(\tilde{t}))+\bar{u} x(\tilde{t})
$$

results, using $f(x(\tilde{t}))-r x(\tilde{t})=f\left(x_{0 \bar{u}}\right)-r x_{0 \bar{u}}$, in

$$
(1-\lambda(\tilde{t}))(f(x(\tilde{t}))-r x(\tilde{t}))=(1-\lambda(\tilde{t})) \bar{u} x(\tilde{t}) .
$$

This can only be achieved if $\lambda(\tilde{t})=1$, which corresponds to a switching instant (and a contradiction) or $(f(x(\tilde{t}))-r x(\tilde{t}))=x(\tilde{t})$, which would mean that $x(\tilde{t})=\bar{x}^{\bar{u}}$, which also is impossible since, in that phase, $x(t)$ was converging in infinite time toward $\bar{x}^{\bar{u}}$ (and $\left.x_{0 \bar{u}}<\bar{x}^{\bar{u}}\right)$. We then conclude that, as announced, $f\left(x_{\bar{T}}\right)-r x_{\bar{T}}>f\left(x_{0 \bar{u}}\right)-r x_{0 \bar{u}}$.

We then use the constancy of the Hamiltonian in both to show that $x_{\bar{u} 0}<x_{0 \bar{u}}$. In the bright phase, we have

$$
\lambda_{0}\left(f\left(x_{0}\right)-r x_{0}\right)=\left(f\left(x_{0 \bar{u}}\right)-r x_{0 \bar{u}}\right),
$$

and, in the dark phase

$$
\lambda_{0}\left(-r x_{0}\right)=\left(-r x_{\bar{u} 0}\right) .
$$

Taking the difference between these equalities yields $\lambda_{0} f\left(x_{0}\right)=\left(f\left(x_{0 \bar{u}}\right)-r x_{0 \bar{u}}\right)+$ $r x_{\bar{u} 0}$. Finally we note that, after $t_{\bar{u} 0}$, the dynamics become $\dot{\lambda}=r \lambda$, so that, using $\lambda\left(t_{\bar{u} 0}\right)=1$ and $\lambda(T)=\lambda_{0}$, we have $\lambda_{0}=\mathrm{e}^{r\left(T-t_{\bar{u} 0}\right)}$, which yields $f\left(x_{0}\right) e^{r\left(T-t_{\bar{u} 0}\right)}-$ $r x_{\bar{u} 0}=f\left(x_{0 \bar{u}}\right)-r x_{0 \bar{u}}$. Concavity implies that $f\left(x_{0}\right) e^{r\left(T-t_{\bar{u} 0}\right)}>f\left(x_{0} e^{r\left(T-t_{\bar{u} 0}\right)}\right)=$ $f\left(x_{\bar{u} 0}\right)$, so that the constancy of the Hamiltonian conditions can only be satisfied if

$$
f\left(x_{\bar{u} 0}\right)-r x_{\bar{u} 0}<f\left(x_{0 \bar{u}}\right)-r x_{0 \bar{u}} .
$$

This can only be achieved for $x_{\bar{u} 0}<x_{0 \bar{u}}$, since $x_{\bar{u} 0}<x_{\bar{T}}$ and $f\left(x_{\bar{T}}\right)-r x_{\bar{T}}>$ $f\left(x_{0 \bar{u}}\right)-r x_{0 \bar{u}}>f\left(x_{\bar{u} 0}\right)-r x_{\bar{u} 0}$.

Bang-singular-bang with $\lambda_{0}>1$ :

We will first look at what a singular arc could be. For that, we see that $\frac{\partial H}{\partial u}=$ $(1-\lambda) x$ should be 0 over a time interval which, since a biomass level of $x=0$ does not make sense when optimizing the productivity, amounts to imposing $\lambda=1$. We 
then compute its time derivatives.

$$
\frac{d}{d t}(\lambda-1)_{\mid \lambda=1}=-f^{\prime}(x) h(t)+r .
$$

When $h(t)=0$, that is in the dark phase, no singular arc is thus possible. When $h(t)=1$, this derivative is equal to zero when $x=x_{\sigma}$ defined in (3.8). The singular control is then the control that maintains this equilibrium, that is $u_{\sigma}$ defined in (3.8). This control is positive thanks to Assumption 1-(ii) but is only admissible if $u_{\sigma}<\bar{u}$. No singular control can exist otherwise. In fact, this implies that $\bar{x}^{\bar{u}}<x_{\sigma}<\bar{x}^{0}$ because $\bar{x}(u)$ is decreasing. The case where $u_{\sigma}=\bar{u}$ has been handled through the bang-bang case.

When a singular branch appears in the optimal solution, it has to be kept among local optimality candidates because the second order Legendre-Clebsch condition (Kelley, Kopp \& Gardner Moyer 1967)

$$
\frac{\partial}{\partial u}\left(\frac{\mathrm{d}^{2}}{\mathrm{~d} \tau^{2}} \frac{\partial H}{\partial u}\right)=-f^{\prime \prime}\left(x_{\sigma}\right) \geq 0
$$

is satisfied on the singular arc because of the concavity.

The construction of the solution is very similar to that in the purely bang-bang case. Similarly, a first switch needs to occur in the interval $(0, \bar{T})$. This switch can be from $u=0$ to $u=\bar{u}$ or from $u=0$ to $u=u_{\sigma}$ and should occur with $x \leq x_{\sigma}$ (Proposition 1(ii)). In fact, if a switch first occurs to $u=\bar{u}, x$ then converges toward $\bar{x}^{\bar{u}}$, so that it does not go on or above $x_{\sigma}$, which prevents any other switch before $\bar{T}$ (Proposition 1-(iii)). No singular arc can then appear. These solutions have been handled earlier.

In bang-singular-bang cases, a switch from 0 to $u_{\sigma}$ then directly takes place once $\lambda=1$ at $\left(t_{0 \sigma}, x_{\sigma}\right)$.

From there, $\lambda(t)=1$ and $x(t)=x_{\sigma}$ for some time. This could be until $t_{\sigma 0} \leq \bar{T}$, followed directly by $u=0$ or the singular arc could end at time $t_{\sigma \bar{u}}<\bar{T}$, where a switch occurs toward $u=\bar{u}$; note that the strict inequality is due to the fact that, if we had $t_{\sigma \bar{u}}=\bar{T}$, we would then have $\lambda(\bar{T})=1$ and $\dot{\lambda}>0$ for $t \geq \bar{T}$, so that $u$ directly goes to 0 : no actual switch to $u=\bar{u}$ has taken place. Using the constancy of the Hamiltonian, we can conclude that a direct switch from $u_{\sigma}$ to $u=0$ is not possible at $t_{\sigma 0} \leq \bar{T}$. Indeed, if such an early switch occurred, we would have

$$
\begin{aligned}
& \lambda_{0}\left(f\left(x_{0}\right)-r x_{0}\right)=\lambda_{\bar{T}}\left(f\left(x_{\bar{T}}\right)-r x_{\bar{T}}\right), \\
& \lambda_{0}\left(-r x_{0}\right)=\lambda_{\bar{T}}\left(-r x_{\bar{T}}\right) .
\end{aligned}
$$

Taking the differences between the equalities, we obtain

$$
\lambda_{0} f\left(x_{0}\right)=\lambda_{\bar{T}} f\left(x_{\bar{T}}\right) .
$$

Taking the quotient of the last two equalities then yields

$$
\frac{f\left(x_{0}\right)}{x_{0}}=\frac{f\left(x_{\bar{T}}\right)}{x_{\bar{T}}},
$$

which we have shown earlier be impossible. The optimal solution then leaves the singular arc with $u=\bar{u}$ strictly before $\bar{T}$, and switches to 0 strictly after $\bar{T}$.

From then on, things are unchanged with respect to the bang-bang case.

Solution with $\lambda_{0} \leq 1$ : 
Since $\dot{\lambda}>0$ in $\lambda=1$ in the dark phase, such a solution would mean that harvesting takes place during the whole dark phase because no transition from $u=0$ to $u=\bar{u}$ can take place in this phase (Proposition 1-(i)). Two possibilities then occur: either $u=\bar{u}$ all the time or switches from $u=\bar{u}$ to $u=0$ or $u_{\sigma}$ and then back to $u=\bar{u}$ take place in the interval $(0, \bar{T})$.

In the latter case, the first switch from $u=\bar{u}$ to $u=0$ can only take place with $x>x_{\sigma}$ (Proposition 1-(iii)). Then, when the control $u=0$ is applied for some time, the solution $x(t)$ is increasing. No switch back to $u=\bar{u}$ can then take place before $\bar{T}$ because such a switch would require $x(t)<x_{\sigma}$ (Proposition 1-(ii)), which cannot occur. An optimal solution of this form cannot exist.

We can also show that no strategy in the $(0, \bar{T})$ interval can have the form $u=\bar{u} \rightarrow$ $u_{\sigma} \rightarrow u=0$ or $\bar{u}$. Indeed, since $0<u_{\sigma}<\bar{u}, \bar{x}^{0}>x_{\sigma}>\bar{x}^{\bar{u}}$, the singular arc can only be reached with $u=\bar{u}$ when coming from above it. If the switch that takes place at the end of the singular phase is from $u_{\sigma}$ to $0, x(t)$ will increase and there should be a subsequent switch from 0 to $\bar{u}$ which is impossible with $x(t)>x_{\sigma}$. If the switch that takes place at the end of the singular phase is from $u_{\sigma}$ to $\bar{u}, x(t)$ will decrease all the time between $t_{\sigma \bar{u}}$ and $T$, which is in contradiction with the fact that we had $x(0)>x_{\sigma}$. The only potential optimal control in that family is therefore $u(t)=\bar{u}$ for all times. 